\title{
Alternativas para la comprensión de la pobreza: hogares y capital social en México*
}

\author{
Luis Portales, ${ }^{* *}$ Mariana Gabarrot***
}

\author{
Perfiles Latinoamericanos | Artículo \\ vol. 23 | núm. 45 | 2015 \\ pp. $59-78$
}

\begin{abstract}
Resumen
En décadas recientes, el capital social se ha visualizado como un elemento clave para el desarrollo tanto en discursos académicos como en programas de política social. Sin embargo, la operacionalización del concepto varía de un lugar a otro, lo que genera debates acerca de sus posibles aportaciones tanto teóricas como empíricas. El presente trabajo argumenta que una definición contextual del capital social contribuye a la construcción de una idea más sofisticada del combate a la pobreza, lo que refuerza el cuestionamiento a los enfoques tradicionales de otras investigaciones sobre el tema. Lo anterior se presenta a partir de los resultados de un trabajo de campo etnográfico que se realizó en la ciudad de Monterrey, Nuevo León, México, el cual incluyó entrevistas en profundidad a miembros de hogares que fueron definidos como representativos de distintos niveles de pobreza con base en una encuesta previa.
\end{abstract}

\begin{abstract}
In recent decades, social capital has been a key element within academic discourses and social policy programs. However, the operationalization of the concept varies from one place to another, thus generating debates regarding its likely contributions - both theoretical and empirical- The paper argues that a contextual definition of the term contributes to the construction of a more sophisticated idea regarding poverty alleviation, supporting other academic work, which questions traditional approaches. The latter is shown through the results of ethnographic fieldwork in Monterrey, Nuevo León, México, which included in depth interviews to household members defined as representatives of different poverty levels by a previous survey.
\end{abstract}

Palabras clave: capital social, pobreza multidimensional, capital económico, capital humano, desarrollo, política social.

Keywords: social capital, multidimensional poverty, economic capital, human capital, development, social policy.

* Parte de las reflexiones presentadas en este artículo fueron posibles gracias al apoyo del programa Ciencia Básica del Conacyt para un proyecto sobre migración y estrategias de vida familiar en el estado de Nuevo León.

** Doctor en Ciencias Sociales y profesor investigador en la Universidad de Monterrey.

*** Doctora en Geografía Humana y profesora investigadora del Tec de Monterrey, Campus Monterrey. 


\section{Introducción}

E

capital social conceptualiza el valor de las relaciones y las redes sociales para acceder a determinados recursos, para crear una diferencia respecto del capital económico — dinero, bienes materiales - y del capital humano conocimiento- (Bourdieu, 1986; Coleman, 1988; Putnam, 1993; Portes, 1998). En la década de 1990 , el capital social se comenzó a visualizar como un elemento clave en la solución de problemas relacionados con el desarrollo y el combate a la pobreza, especialmente por parte de organismos internacionales, tales como el Banco Mundial y el Programa de Naciones Unidas para el Desarrollo (PNUD) (Durston, 2003; Siles et al., 2003; Robison et al., 2003; Narayan, 1999; Millán, y Gordon, 2004; Portes, y Landolt, 2000; Woolcock, 2001). Dada su naturaleza relacional, el capital social varía de un contexto a otro y su articulación con otros capitales es dinámica, lo cual dificulta el establecimiento claro de sus aportaciones teóricas y empíricas.

En este contexto, el presente trabajo muestra cómo una definición precisa del término contribuye a entender la forma en que el capital social de hogares en pobreza es movilizado para acceder a recursos, lo que aporta elementos para una reflexión más detallada acerca de las políticas de desarrollo. En general, se pone en claro que este capital ayuda en la construcción de una idea más sofisticada del combate a la pobreza, con lo cual contribuye a una literatura que ha cuestionado el enfoque del ingreso como el mejor para entender o solucionar este fenómeno (Rojas, 2010).

El texto se ha dividido en cinco secciones. La primera realiza un análisis teórico acerca de las características del capital social y de los elementos que lo conforman. La segunda discute su uso en la medición de la pobreza multidimensional desarrollada por el Consejo Nacional de Evaluación de la Política Social (CONEVAL). La tercera y cuarta secciones ofrecen una propuesta metodológica además de material empírico sobre el manejo de los tres tipos de capital en hogares de una colonia marginada en Monterrey, Nuevo León. A modo de conclusión, se discuten los resultados de este trabajo, así como algunas implicaciones para la reflexión teórica del capital social y su influencia en la práctica del combate a la pobreza.

\section{Sobre el capital social}

Las aproximaciones al capital social coinciden en que éste se conforma de las relaciones sociales recíprocas, de las redes sociales, y de la capacidad que estos 
dos componentes tienen de generar algún tipo de beneficio para un actor específico. Las relaciones sociales pueden ser clasificadas a partir de varios criterios: fuerza del lazo entre dos o más personas — fuerte o débil— (Granovetter, 1973), motivaciones que producen esas relaciones (Wellman, y Wortley, 1990), papel de los actores (Woolcock, 1998), función y tiempo de las relaciones sociales (Nahapiet, y Ghoshal, 1998), entre otros. Las redes, por otra parte, tienen que ver con el número y estructura de las relaciones sociales (Fuhse, 2009). En una red cerrada, donde la mayoría de los actores se conocen entre sí, se refuerza la homogeneidad del grupo y su identidad (Putnam, 1995; Svendsen, y Haase Sevendsen, 2009; Woolcock, 1998), mientras que en una más abierta se permite la conexión entre actores de diferentes perfiles (Burt, 2005).

Es importante recordar que el capital social es un medio de intercambio, por lo que da acceso a otros capitales como el económico o el humano (Bourdieu, 1986), lo que le brinda el potencial de ser un elemento positivo en la mejora de las condiciones de vida de grupos que se encuentran en situación de vulnerabilidad. Sin embargo, la reciprocidad implícita también puede ser una carga en la esfera individual, por ejemplo, cuando una persona es agobiada por la demanda de favores proveniente de familiares y amigos en determinado momento. Por otro lado, algunos grupos sociales con reservas importantes de capital social pueden acarrear repercusiones negativas en la dinámica de ciertas comunidades (Portes, y Landolt, 1996, 2000; Vigil, 2003); el ejemplo más utilizado en este sentido es el de las mafias. No obstante, el aspecto positivo del capital social ha sido entendido como un mecanismo clave en el combate a la pobreza (Narayan, 1999; Portes, y Landolt, 2000; World Bank, 2006).

A grandes rasgos, la pobreza implica que hay una condición óptima de vida de la cual se ha excluido a determinados individuos o grupos y, según el enfoque en que aquélla sea entendida, podemos comprender su relación con el capital social. Así, si se considera la pobreza desde una perspectiva monetaria, su conceptualización se dará en función de los ingresos de un individuo y el capital social juega un papel funcional. Por lo tanto, su construcción o movilización permite solamente incrementar recursos (Bebbington, 2005; Durston, 2003; Robison, Siles, y Schmid, 2003). En contraste, si la pobreza se entiende desde la perspectiva de la falta de capacidades y de la calidad de vida, entonces el capital social forma parte de la propia definición, es decir, su carencia denota una situación de exclusión o privación que reduce las condiciones de vida y evidencia un contexto de pobreza (Sen, 1981; Narayan, 1999; Noll, 2010; Stiglitz, Sen, y Fitoussi, 2008). Esta multiplicidad de opciones para abordar la relación capital social-pobreza refleja la complejidad conceptual a la cual nos enfrentamos al momento de su aplicación empírica. La siguiente sección discute cómo se han discutido estos dilemas en el caso particular de la realidad mexicana. 


\section{La pobreza multidimensional en México}

El parteaguas en la medición de la pobreza en México fue la creación de la Ley General de Desarrollo Social (LGDS, 2004) que, en el marco de la definición de los derechos sociales y la creación del Consejo Nacional de Evaluación de la Política Social (ceneps) (Boltvinik, 2005; Cortés et al. 2002; Townsend, 2003), la definió como un fenómeno multidimensional padecido por quienes "no tienen garantizado el ejercicio de al menos uno de sus derechos [...] y que sus ingresos son insuficientes para adquirir los bienes y servicios que requiere para satisfacer sus necesidades" (CONEVAL, 2010: 20). Esta definición busca acercarse a la idea de capacidades y calidad de vida a través de tres espacios analíticos: 1) enfoque de bienestar, 2) derechos fundamentales, y 3) cohesión social.

El enfoque de bienestar utiliza una aproximación económica, para la cual se construyeron dos referencias: a) Línea de Bienestar Mínimo (LBM) —la población por debajo de esta línea no cuenta con recursos suficientes para satisfacer sus necesidades alimentarias-, y b) Línea de Bienestar (LB) —la población por debajo de esta línea puede satisfacer sus necesidades alimentarias, pero no puede adquirir suficientes bienes y servicios.

Los derechos fundamentales identifican a la población con al menos una carencia social en los indicadores asociados con esta idea: rezago educativo, acceso a los servicios de salud, acceso a la seguridad social, calidad y espacios de la vivienda, acceso a servicios básicos en la vivienda, y acceso a la alimentación. De estos dos espacios analíticos se han establecido cuadrantes de acuerdo con el ingreso y las privaciones sociales. En el primer cuadrante (I) se encuentran los pobres por ingreso y por carencias, incluyendo los pobres extremos o pobres alimentarios. Los siguientes dos presentan a personas vulnerables (II y III), ya sea por insuficiencia de ingresos o por carencias en términos de derechos. El último cuadrante (IV) presenta a la población no pobre (CONEVAL, 2010).

En la sección que sigue se presenta la medición de pobreza multidimensional del CONEval para analizar cómo el capital social se articula con los capitales económico y humano de los hogares de una colonia marginada del área metropolitana de Monterrey (АмM), Nuevo León.

\section{Pobreza y capital social en la colonia Fernando Amilpa de Monterrey}

El AMM se conforma de nueve municipios, mismos que en términos generales presentan índices de rezago social muy bajos. A pesar de ello, más del 27\% de la población se encuentra en alguna situación de pobreza (CONEVAL, 2012) 
y en algunas zonas existen índices de rezago social alto o muy alto, los cuales son similares a los de otras zonas urbanas marginadas del país e inclusive de América Latina (Ziccardi, 2008). En este sentido, el perfil de la colonia Fernando Amilpa no es muy distinto al de otras del país: cuenta con alrededor de 1300 hogares, de los cuales 58\% muestran índices de rezago social considerados muy altos y 42\% altos (Martínez, Treviño, y Gómez, 2009).

En 2011, la colonia Fernando Amilpa contaba con planteles de educación preescolar, primaria y secundaria, un centro de salud y un Centro Comunitario de Desarrollo Social (CCDs), el cual, administrado por el gobierno estatal, funge como eje de la política social, concentrando su labor en la realización de talleres de capacitación para niños, jóvenes y adultos. Asimismo, la mayor parte de los hogares — alrededor del 80\% — contaba con cobertura de servicios básicos (luz, drenaje, pavimentación en su calle y agua). Si bien los resultados descritos son específicos de este caso, también pueden servir de punto de partida para establecer paralelos interesantes.

A fin de comprender la forma en que el capital social se articula con el capital económico y humano, en esta investigación se ha adoptado una metodología mixta. Se realizó observación participante —en la que el autor principal trabajó como facilitador voluntario en los CCDs de la colonia- por un periodo que abarcó de marzo de 2010 a junio de 2011. Asimismo, se llevaron a cabo entrevistas estructuradas a una muestra aleatoria de 96 hogares, de un universo de 1431 , lo que permite una confianza de $95 \%$ y un error de $0.1 \%$. Se utilizó como base el cuestionario CONEVAL para determinar los niveles de pobreza en los hogares, de acuerdo con la metodología de dicho organismo, y se incluyeron además aspectos vinculados con sus relaciones y redes sociales. Al momento de aplicar el cuestionario, se preguntó a los hogares acerca de relaciones de amistad y parentesco que les pudieran dar acceso a recursos específicos. De este modo se documentó un perfil de posibles articulaciones de capital social para cada uno de los hogares. Posteriormente, se seleccionaron cuatro hogares en pobreza extrema, tres en pobreza multidimensional, tres en situación de vulnerabilidad —ingresos y carencias—, y se tomó en cuenta que fueran relativamente homogéneos en función de sus ingresos, carencias sociales y tipos de relaciones sociales para aplicarles entrevistas en profundidad. ${ }^{1}$

En dichas entrevistas se abordaron aspectos relativos al capital económico, humano y social de los hogares (cuadro 1). Para la conformación del capital económico se consideraron las dimensiones de ingresos y calidad de la vivienda

1 Se decidió llevar a cabo una entrevista más en el perfil de pobreza extrema, dada la complejidad de la realidad social que estos hogares enfrentan, tal como se aprecia en este artículo. Todos los hogares se pueden considerar adultos o jóvenes (los generadores de ingreso tenían entre 40 y 25 años). 
(Rodríguez y Kinto, 2010), mientras que para el capital humano se consideró la educación y el acceso a la salud (Sen, 1981). En cuanto al capital social, tal y como se mencionó en la primera sección, se utilizaron los dos componentes que lo conforman: relaciones y redes sociales.

Cuadro 1. Elementos considerados en las entrevistas en profundidad por tipo de capital

\begin{tabular}{lll}
\hline Capital & $\begin{array}{l}\text { Elemento } \\
\text { considerado }\end{array}$ & Aspectos cubiertos \\
\cline { 2 - 3 } Económico & Ingresos & $\begin{array}{l}\text { Actividades productivas formales realizadas por el hogar que conforman la base de su gasto. } \\
\text { Actividades productivas informales realizadas por el hogar para contribuir al gasto. }\end{array}$ \\
\cline { 2 - 3 } & Vivienda & $\begin{array}{l}\text { Materiales utilizados en la construcción. } \\
\text { Historia de la construcción de la vivienda. } \\
\text { Condiciones de hacinamiento del hogar. }\end{array}$ \\
\hline \multirow{2}{*}{ Humano } & Educativa & $\begin{array}{l}\text { Asistencia de los miembros de la familia a la escuela; motivaciones para seguir o } \\
\text { desertar de ella. } \\
\text { Participación en capacitaciones y talleres fuera del sistema educativo. }\end{array}$ \\
\cline { 2 - 4 } & Salud & $\begin{array}{l}\text { Lugar donde regularmente se atienden los miembros del hogar. } \\
\text { Percepción sobre la atención que reciben los miembros del hogar en materia de salud. }\end{array}$ \\
\hline \multirow{2}{*}{ Social } & $\begin{array}{l}\text { Relaciones } \\
\text { sociales }\end{array}$ & $\begin{array}{l}\text { Relaciones que tienen los miembros del hogar y que permiten acceder a cualquiera de } \\
\text { los aspectos cubiertos por el capital económico y humano. }\end{array}$ \\
\cline { 2 - 4 } & $\begin{array}{l}\text { Redes } \\
\text { sociales }\end{array}$ & $\begin{array}{l}\text { Redes producto de las interacciones existentes entre las relaciones sociales que tienen } \\
\text { los miembros del hogar. }\end{array}$ \\
\hline Fuente: Elaboración propia. &
\end{tabular}

En las entrevistas, mediante la reconstrucción de la historia del hogar, se abordó cada uno de los capitales por separado y se buscó la relación que éstos tenían entre sí. Por ejemplo, al momento de hablar sobre la vivienda, no solo se identificó la historia seguida hasta llegar a su situación actual, sino que además se tocó el tipo de conocimiento que necesitaron para realizar dicha construcción y las personas ajenas al hogar que habían participado en su realización. La forma en que se presentarán los resultados será a través del vínculo del capital social con los elementos considerados en el capital económico y humano de los hogares.

\section{Tipos de capital social en la colonia Fernando Amilpa}

El análisis de las entrevistas en profundidad permitió detectar cómo se construye y sostiene el capital social por medio de distintos tipos y formas de interacción. Se identificó la importancia de la cercanía que existe entre los integrantes del hogar, y los papeles sociales que juegan con quienes establecen las relaciones (Putnam, 1995; Woolcock 1998). Sin embargo, existen otros elementos referidos a la cercanía afectiva y cognitiva que cada actor social tiene al establecer 
una relación particular, además de la importancia del contexto o motivación por la cual aquélla ha surgido.

Por lo anterior, se decidió proponer una nueva categorización que retome las diferentes relaciones sociales, pero que además considere dos aspectos cruciales. El primero de éstos es el tipo de cercanía al que hicieron referencia los miembros del hogar en términos de la frecuencia, el espacio de interacción donde se lleva a cabo y la cercanía cognitiva que tiene la relación social. Esto ayudó a identificar la naturaleza de los lazos desde un punto de vista afectivo y funcional. El segundo aspecto fue la tendencia que estos vínculos presentaban en el grado de apertura o cierre de la red social.

De esta forma, hemos construido tres tipos de capital social: fraternal, colaborador y corporativo, cada uno de los cuales se corresponde con distintos grados de afectividad, función y estructura de la red (cuadro 2). Por ejemplo, un hogar con relaciones sociales que muestran una alta cercanía afectiva, como son hermanos o amigos cercanos, tiende a generar redes más cerradas que aquellas relaciones que se tienen con líderes sindicales, en donde la tendencia es a construir redes más abiertas. ${ }^{2}$

Cuadro 2. Capital social de acuerdo al tipo de relaciones establecidas por el hogar

\begin{tabular}{lll}
\hline $\begin{array}{l}\text { Tipo de capital } \\
\text { social }\end{array}$ & Relaciones sociales & Redes sociales \\
\hline \multirow{2}{*}{ Fraternal } & $\begin{array}{l}\text { Familiares y amigos que se frecuentan al menos } \\
\text { una vez a la semana y se visitan en sus casas. } \\
\text { Surge por un vínculo consanguíneo o de amistad. }\end{array}$ & $\begin{array}{l}\text { Cerrada. Los integrantes de la red se cono- } \\
\text { cen e interactúan entre sí. }\end{array}$ \\
& $\begin{array}{l}\text { Familiares políticos y conocidos con quienes se } \\
\text { Colaborador } \\
\text { tiene contacto mensual u ocasional. Surgen por }\end{array}$ & $\begin{array}{l}\text { Semiabierta. Los integrantes se conocen, la } \\
\text { cercanía territorial suele ser importante, pero } \\
\text { permite la existencia de huecos estructurales. }\end{array}$ \\
& $\begin{array}{l}\text { una relación familiar política. } \\
\text { Colaciones de tipo clientelar con funcionarios }\end{array}$ & $\begin{array}{l}\text { Abierta. La mayor parte de los integrantes no } \\
\text { se conocen entre sí. }\end{array}$ \\
\hline
\end{tabular}

Fuente: Elaboración propia.

Esta descripción permite aproximarnos a la aprehensión de características específicas del funcionamiento del capital social y de las relaciones concretas que puede tener con el resto de los capitales. Si bien esto significa un reto por su complejidad, en realidad abre la posibilidad de estrechar la relación entre nuestra conceptualización del capital social y las propuestas que pueden hacerse en términos de su relación con la pobreza. La siguiente sección presenta este ejercicio analítico.

2 Esto no quiere decir que no se pueda presentar el caso de relaciones fraternales abiertas; sin embargo, la probabilidad de que esto suceda, según lo encontrado en las entrevistas y el trabajo de campo, es muy baja. 
Este tipo de capital social se integra de las relaciones que el hogar mantiene con familiares cercanos y amigos, las cuales tienden a construir redes cerradas en donde todos los integrantes se conocen y observan un alto grado de interacción. Respecto a la principal fuente de ingresos del hogar, se encontró que el uso de este capital social prevalece en los hogares en pobreza extrema, lo que favorece la transmisión de oficios de generación en generación (recolectores de basura o albañiles), los cuales carecen de prestaciones sociales y perciben bajos ingresos.

Un ejemplo es el de doña Teresa, quien se encuentra en pobreza extrema. La recolección de la basura la comenzó su esposo en compañía de sus hermanos, y ahora de su hijo de diecisiete años. Ella misma lo explica: "[mi esposo] tenía el carretón de un hermano [...] andaban trabajando en la basura [...] juntan cartón, botes para vender [...] mi hijo ahora anda juntando cartón [...] los dos [padre e hijo] andan recogiendo de la basura".

Este capital social no solo contempla relaciones familiares, sino otras de amistad, que ayudan a la obtención de trabajos precarios. Tal situación la vive Mercedes, jefa de un hogar en situación de pobreza extrema, quien narra cómo ha conseguido trabajo gracias a las amigas de su colonia: "yo conseguía trabajo ahí con las amigas [...] de la misma colonia [...] no teníamos prestaciones ni nada, pero pues queríamos trabajar”. En este hogar se observa una vez más la transmisión de los oficios precarios de generación a generación; la pareja actual de Mercedes, Humberto, aprendió el oficio de la recolección de basura por su madre: "Mi mamá tiene carretón y yo le ayudaba [...] y pues de ahí aprendí el negocio".

De esta forma se observa cómo, a pesar de que la relación que propició la realización de cierto tipo de trabajo es diferente — familiar o amigo-, el común denominador es el capital social fraternal y la precariedad del trabajo al que brinda acceso.

A diferencia de los hogares en pobreza extrema, en los de pobreza multidimensional el capital social fraternal se utiliza como mecanismo de financiamiento que encuentra su forma más tradicional en la organización de tandas, ${ }^{3}$ que son utilizadas para la mejora de la vivienda. Éste es el caso de dońa Flora, quien organizaba a sus vecinas para realizar tandas que le permitieran construir su casa: “iMi casa fue hecha con puras tandas! [...] aquí organizaba a las vecinas

3 Mecanismo de ahorro basado en la confianza y reciprocidad de los miembros de la red social, donde todos los miembros aportan una cantidad cada determinado tiempo y el acumulado se destina a un miembro del grupo según el turno que se le haya asignado. La tanda finaliza cuando todos han recibido en alguna ocasión el fondo acumulado. 
o a las comadres, hacíamos tandas de mil a dos mil pesos [...] ahí poco a poco iba juntando y echando cada vez más a la casa”.

Las tandas solo se hallaron en los hogares en pobreza multidimensional, situación que tal vez se deba a que los hogares en pobreza extrema temen no contar con los recursos suficientes para pagarlas. Esto lo expresa Camilo, quien pertenece a un hogar en pobreza extrema y que debido a la precariedad del trabajo prefiere no participar en ellas: "Eso de las tandas no muy me gusta [...] por lo mismo de que no sé cuánto voy a sacar en el día, pos mejor no [...] así uno no queda mal con las personas". Por otro lado, los hogares en situación de vulnerabilidad no precisan tandas, dado que tienen acceso a créditos por parte de instituciones privadas. Esto lo menciona Gloria quien presenta una situación de vulnerabilidad por ingresos y obtiene préstamos de diferente índole: "Tengo que pagar todo, la cuenta del banco, lo que debía de Famsa de mi lavadora; todavía lo estoy pagando y no termino de pagar [...] estamos ahorita hasta el cuello".

El capital social fraternal permite además la realización de mejoras de la vivienda, como pueden ser la construcción de techo de concreto, la colocación de piso firme o la edificación de un cuarto. Doña Esther, perteneciente a un hogar en situación de pobreza multidimensional, recuerda cómo sus hijos acudían a ayudarle al padre, aun cuando ya no habitaban en el hogar: "Ahí entre los tres [padre e hijos] la hicieron [la casa] [...] Primero fueron los cuartos y luego de un tiempo los pisos; duramos mucho sin pisos, hasta que volviéramos a juntar para comprar, y asî".

Asimismo, este capital social tiene impactos positivos en la educación, sin importar el tipo de pobreza. Por ejemplo, los miembros que alguna vez pertenecieron al hogar y que no pudieron terminar la escuela secundaria o incluso la primaria, aportan al gasto educativo o a la manutención escolar de alguno de los miembros más jóvenes, independientemente de su condición social. Eréndira recuerda cómo ella y sus hermanos mayores, aun y cuando ya no vivían en la casa de sus padres, apoyaron a sus hermanos menores con su educación: "Nosotros tres [hermanos mayores] estudiamos hasta la primaria [...] Nada más las más chiquitas fueron las que terminaron la secundaria [...] nosotros empezamos a aportarle a mi mamá dinero para que ellas siguieran".

Sin embargo, sólo los hogares vulnerables utilizaron este mismo capital social para acceder a la educación informal — aquella que adoptan los miembros del hogar y que no pertenece al sistema educativo, tales como cursos y capacitaciones-. Por ejemplo, Gloria, además de acceder a diferentes créditos, narra cómo gracias a la relación con su mejor amiga y comadre, tomó varios cursos: "Ella [mi comadre] estudiaba belleza y yo corte y confección ahí en el centro [de Monterrey]. No le dijo, donde ella iba, yo iba”. 
En cuanto a la salud, el capital social fraternal funciona como un mecanismo de acceso. Esta situación se presenta cuando algún familiar cuenta con seguro social o popular y lo extiende a familiares consanguíneos, aun cuando éstos no habiten en el hogar. Octavio, que se encuentra en pobreza multidimensional, narra la situación en la que se encuentra su suegra, quién habita en la misma vivienda y recibe protección social por medio de sus hijos: "Ella tiene acceso al seguro social [...] ella no trabajó y pues no tenía ni cómo [...], son sus hijos quienes la afilian, ellos sí están inscritos por parte de su trabajo [...], ahí la afiliaron a ella".

Esta situación se relaciona con el tipo de actividad productiva formal con la que generan sus ingresos. En este caso, los hogares en situación de pobreza multidimensional y extrema no reciben protección social (75 y $72 \%$, respectivamente) por parte del empleador, mientras que los hogares en situación de vulnerabilidad sí la reciben (85.2\%) o tienen la posibilidad económica de acceder a la salud por sus propios medios.

En general, se observa que el capital social fraternal se articula de diversas formas con el capital económico, según el tipo de pobreza de los hogares. Por ejemplo, para el caso de los hogares en situación de pobreza extrema, este capital incide en la repetición de oficios de una generación a otra. Sin embargo, para el caso de los hogares en pobreza multidimensional, éste es un mecanismo de financiamiento. Es interesante observar que independientemente de la situación de pobreza, dicho capital presenta impactos positivos en términos de acceso y mejora de la vivienda. Y en el ámbito educativo favorece la permanencia de los miembros más jóvenes en la escuela, situación que no se mostró relacionada con el tipo de pobreza. Finalmente, permite el acceso al sistema de salud a familiares consanguíneos. En contraste, el capital social colaborador no se encuentra relacionado con el acceso a la salud o a la educación, pero sí lo está con el capital económico.

\section{Capital social colaborador}

El capital social colaborador se conforma de las relaciones entre familiares políticos y conocidos, con los cuales no se tiene un lazo afectivo muy fuerte, lo que permite la creación de una red social más abierta en la cual los integrantes pueden o no conocerse entre sí.

Respecto al acceso a fuentes de ingresos económicos, los hogares en pobreza multidimensional y con algún tipo de vulnerabilidad suelen usar este capital para conseguir mejores empleos. Es el caso de Salomón, jefe de familia de un hogar en vulnerabilidad por ingresos, que comenzó a trabajar desde niño re- 
partiendo periódico con su papá — capital social fraternal—, pero que gracias a conocidos y vecinos logró un mejor empleo, en donde cuenta con mayores prestaciones sociales:

Siempre he trabajado [...], a la escuela no iba, vendía periódico junto con mi papá y mis hermanos [...] Buscaba más prestaciones porque no tenía ninguna [...] conocidos me decían que en tal parte están buscando y ahí iba [...] ahorita tengo caja de ahorro, aguinaldo, bono de despensa, más uno que le llaman retroactivo.

Este capital social también se relaciona con la realización de actividades productivas efectuadas con el interés de incrementar los ingresos del hogar y que pueden ser venta de productos por catálogo, de tamales, de ropa usada, entre otros. En este sentido, Gloria — esposa de Salomón— muestra cómo merced al establecimiento de relaciones con vecinos y conocidos incrementaba el número de clientes potenciales e ingresos:

A donde iba llevaba mi máquina de coser y yo cosía la ropa [...] Le cosía a la gente de ahí mismo [...] se enteraban por mi suegra, mis cuñadas, porque ellas me daban ropa para coser y pos les decían [...] vendía empanadas casa por casa por toda la colonia, y así me fui haciendo de conocidos.

En relación con la vivienda, el capital social colaborador funciona para que los hogares accedan a materiales o construcción de la vivienda, situación observada principalmente en hogares en pobreza extrema. Esta condición puede tener su origen en el hecho de que estos hogares sufren mayores carencias, lo que los hace susceptibles a recibir alguna ayuda por parte de conocidos, tal como refleja Mercedes, quien debido a su situación de pobreza extrema recibió ayuda de conocidos para la construcción de su cuarto:

No tenía quién me hiciera el cuarto [...] el muchacho de enseguida [...] él me ayudó, él y sus hermanos [...] el esposo de mi cuñada en la fábrica donde trabaja, la tarima que no quieren la queman o la tiran, y él me empezó a acarrear tarima [...] y lo hice de tarimas [el cuarto].

En términos de capital humano, este capital social fungió como un mecanismo de comunicación con el cual los miembros del hogar se informaron sobre la oferta de cursos de capacitación o bien de programas sociales, como es el caso del dedicado al adulto mayor. Esta situación la vivió dońa Tere, quien recibió información por parte de sus vecinos sobre la forma de acceder al Programa de Apoyo Directo al Adulto Mayor: "Estaba una señora 
también [inscrita al programa] [...] [Vive] aquí enfrente [...] Se fueron a apuntar, ellos ya tienen tarjeta $[\ldots]$ un señor grande y una señora $[\ldots]$ me falta apuntar a mi señor”.

En general, el capital social colaborador se relaciona con el económico, principalmente según el tipo de pobreza en que se encuentren los hogares. Aquellos con menor nivel de pobreza son los que han utilizado este tipo de capital para mejorar sus fuentes de empleo. Mientras que para los más pobres, éste representa una mejora en su vivienda. Situación similar fue vista para el capital social corporativo, el cual fungió como un mecanismo de comunicación para acceder a programas sociales relacionados con la salud, pero que jugó un papel importante en el acceso a la salud y la vivienda.

\section{Capital social corporativo}

El último tipo de capital social es el corporativo. Está compuesto por las relaciones que el hogar sostiene con actores institucionales y organizacionales; relaciones que, en general, se encuentran inmersas en una red abierta, en donde los miembros difícilmente se conocen a fondo. El capital social corporativo no mostró un fuerte vínculo con el tipo de ingresos, aunque ello se presenta de forma coyuntural cuando el actor que pertenece a una organización conoce alguna situación de vulnerabilidad o pobreza y ofrece alguna ayuda.

Esta situación la ejemplifica Mercedes, a quien ayudaron para construir su vivienda de tarimas y láminas, y que recibió una oferta laboral por parte de uno de los encargados de la penitenciaría: "vino un señor del penal [penitenciaría] [...] quería que fuera a enseñar a todas las que están encerradas ahí y yo me apunté, y haz de cuenta, salió [el trabajo]".

El capital social corporativo juega un papel importante en la obtención de algún terreno para construir la vivienda. Tal situación no está supeditada al tipo de pobreza, sino a la capacidad de los miembros del hogar para acceder y usar este tipo de capital. Existen varias formas con las que los hogares acceden a la vivienda. La primera es la que presenta la misma Mercedes, quien a pesar de su situación de pobreza extrema se hizo de un terreno por medio de la relación directa con la gente de gobierno:

Yo vivía allá abajo [...] ya nos iban a sacar, iban las patrullas a sacarnos y a tumbarnos los cuartos [...] Lalo [perteneciente a la presidencia municipal] siempre me vio a mí sola allá abajo, entonces cuando él se vino a reubicar para acá me dio una tarjetita que decía lote y manzana [ubicación del terreno] [...] pues ya me quedé con el terreno. 
La segunda forma la representa doña Flora, que pertenece a un hogar en situación de pobreza multidimensional y que logró obtener su casa gracias a su relación con los líderes sindicales que reubicaban las personas que se encontraban en terrenos irregulares:

Estaban solos los terrenos [...] una vez hubo reubicación y la gente agarró, yo era una de las tres que andaban con los líderes [sindicales] [...] medía los terrenos $[\ldots]$ medí tres y con los tres me quedé $[\ldots]$ nada más agarré uno porque pensé que más gente necesitaba [...] [Los otros dos] eran de mis hijos.

Dado que la naturaleza de esta clase de articulación con el capital económico es coyuntural, no se relaciona con algún tipo de pobreza específica.

En la dimensión educativa, el capital social colaborador permite la obtención de favores: una prórroga en el pago de las cuotas escolares, o conseguir que se permita que los hijos utilicen material didáctico que no es el estipulado por la escuela. Esta situación sólo se registró en los hogares vulnerables, como el de Gloria, quien a través de su relación con la maestra y la directora de la escuela logró que sus hijos asistieran a la escuela y cumplieran con sus obligaciones:

No son los útiles que les pidieron en la escuela [...] hablé con la maestra y le expliqué [...] me dijo: "no, con eso tráigaselo" [...] Para que me dieran tiempo para pagar las cuotas fui a hablar con la directora [...] no me dijo nada [...] ella sabe que cada ańo que pasa, yo puntualmente pago.

Este mismo capital social permite que los miembros del hogar ingresen a algún curso, capacitación o taller de diversa índole — desarrollo personal, artístico o productivo- - El hecho se presentó principalmente entre los hogares en situación de pobreza extrema y multidimensional.

Eréndira, en situación de pobreza extrema y que ayudó a que sus hermanos concluyeran su educación escolar, explica cómo inició su proceso de participación en estas actividades y cómo se ha involucrado cada vez más: "a participar aprendí en Caritas [...] me enteré por anuncios, iba a dejar a la niña a la escuela y me entregaron volantes [...] [Después] entré como promotora [...] daba pláticas de drogadicción, nos enseñan a nosotras y luego nosotras enseñamos".

Para la consolidación de esta educación informal impactó el CCDs, impulsado por la Secretaría de Desarrollo Social del Estado de Nuevo León. Este centro surgió con la intención de organizar y crear comités de participación social mediante los cuales se diera respuesta a las necesidades percibidas por los habitantes de la colonia: educación, salud, infraestructura, entre otros. 
Gloria formó parte de estos primeros comités y narra cómo fue el proceso de consolidación:

cuando empezó [el centro comunitario] éramos como unas seis en la cuadra [...] en ese entonces había bastantes grupitos que andaban así [...] teníamos un horario para cuando nos juntábamos ahí [en el centro comunitario] [...] había otro horario también para otros grupos [...] tratábamos [temas relacionados con] la vigilancia, las calles, la luz, el agua, todo [...] ellos [la gente del centro] nos decían: "tienen que hacer su hoja, su oficio", yo me encargaba de hacerlo [...] Nos llevaban y nos decían, vayan ahí, entren y pregunten por tal, y así le hacíamos.

En cuanto al acceso a la salud, el capital social colaborativo solamente funcionó como mecanismo de comunicación, pues, por su medio, hogares con alguna situación de vulnerabilidad — hijo con discapacidad, embarazo prematuro o personas de la tercera edad - conocieron los diferentes programas que el gobierno ofrece. Un ejemplo es el caso de dońa Tere, quien tiene un hijo de ocho años (Rolando) con síndrome de Down. Ella se enteró de un programa de apoyo para nińos con discapacidad del municipio de Escobedo gracias a una persona que trabaja en la administración municipal y que vio a Rolando en un mitin político: "Vino una señora [...] trabaja en Escobedo [...]. Me vio un día con él [Rolando] y me dijo que le llevará la papelería [...] llevé la papelería y apunté al niño". En este caso, la cercanía territorial propicia que los funcionarios identifiquen posibles beneficiarios del programa.

Así, se puede decir que este capital social cumple una doble función: por un lado permite el acceso de los miembros del hogar a diferentes tipos de educación, y por el otro ha sido un medio por el cual las organizaciones e instituciones llevan a cabo sus funciones.

\section{Pobreza multidimensional y capital social en el ámbito local}

Una vez que se ha presentado cómo se relacionan los tipos de capital social con las dimensiones del capital económico y humano, se puede advertir similitudes y diferencias ilustrativas en términos de su articulación. En primera instancia, se observa que todos los hogares, sin importar la clase de pobreza que presenten, cuentan con todos los tipos de capital social identificados, y se diferencian entre sí por la forma en que cada uno de ellos es movilizado según el capital al que se desea tener acceso. Cómo se determinan estas prioridades es el resultado de la dinámica interna de cada hogar y no afecta el hecho de que los distintos tipos de capital social se utilizan de forma estratégica en función 
de los otros capitales — tanto humano como económico (Durston, 2003; Lin, 1999; Woolcock, 1998).

En esta línea, el capital social fraternal surge como la principal fuente de ingresos económicos de los hogares que se encuentran en pobreza extrema, pues gracias a él acceden a fuentes de empleo. Esto confirma la importancia de las redes sociales para la sobrevivencia de los hogares en situación de pobreza extrema (Lomnitz, 2006), pero también el papel que juegan en la permanencia y repetición de patrones de precariedad laboral, mismos que se transmiten de generación a generación, lo que favorece la permanencia de la pobreza con el paso del tiempo (Lewis, 1961; Nélida, y Rocchi, 2007). Este aspecto contrasta con lo identificado en los hogares en pobreza multidimensional y vulnerabilidad, donde existe la posibilidad de mejora en las fuentes de empleo, la cual se potencializa por medio del capital social colaborador.

Lo anterior implica que el ingreso no se relaciona sólo con la generación de fuentes de empleo, sino también con los mecanismos de los hogares para acceder a éstas. Tales mecanismos dependen de las relaciones sociales que ostenten los miembros del hogar (Granovetter, 1973). Se podría agregar que hogares en pobreza extrema mostraron un menor acceso a capital social colaborador, o bien, que dicho acceso no alcanza para romper los patrones de trabajo, pues sus conocidos y amigos tienen empleos similares. Si el caso es el primero, entonces los hogares que logren cambiar el capital social por medio del cual acceden a sus fuentes de empleo, tendrán mayor posibilidad de salir de su situación de pobreza. Es decir, el capital social por sí sólo no implica siempre una mejora, además de que es importante diferenciar claramente entre el capital social y los recursos a los que éste da acceso (Portes, y Landolt, 2000).

En cuanto a la adquisición de capital humano, el capital social fraternal y el capital social corporativo dieron muestras de la semejanza que existe entre los hogares en pobreza multidimensional y aquellos en pobreza extrema. Ambos tipos de hogares utilizan el capital social fraternal para acceder a una educación formal y a la salud; mientras que el capital social corporativo permite acceder a diferentes capacitaciones. Mientras tanto, los hogares vulnerables sólo utilizan el capital social corporativo para acceder a la educación formal y el social fraternal para asistir a cursos de capacitación. Por otro lado, el hecho de que los hogares con niveles más elevados de pobreza accedan a la salud por medio de sus familiares — por ejemplo, al otorgarles acceso al seguro social o al populares un síntoma de la exclusión que caracteriza los hogares urbanos en pobreza extrema y multidimensional (Gordon, 2008). Asimismo, ello da muestra de cómo el capital social funciona a manera de herramienta en términos de una estrategia de vida con la que se logra dar respuesta a esta situación de exclusión (Bebbington, 2005). 
Otro aspecto interesante fue observar que el capital social corporativo no brindó acceso a ninguna fuente de ingresos; más bien su vínculo con los capitales económico y humano está dado de forma coyuntural, como lo muestra el caso de la vivienda, las capacitaciones y los favores que permiten a los miembros continuar en la escuela. Además, este capital social surge de la convivencia derivada de la cercanía física, es decir, presenta una lógica territorial. En este sentido, se puede suponer que la geografía, vista como un espacio de interacción social, juega un papel importante en la creación del capital social corporativo. Este espacio permite a los funcionarios públicos establecer puentes de comunicación y diálogo, mediante los cuales se da respuesta a las necesidades expuestas por los miembros de los hogares que habitan en él. En todo caso, también sería pertinente problematizar estos vínculos en términos del grado de clientelismo que pudieran generar, especialmente cuando de ellos depende el acceso a servicios públicos por parte de poblaciones vulnerables (Portales y Ruiz, 2013).

En general, el análisis microsociológico de los capitales muestra que todos los hogares, sin importar el tipo de pobreza que presenten, cuentan con capitales que son articulados entre sí en aras de mejorar su situación actual, si bien su principal elemento de diferenciación es el modo de articularlos. Esto permite suponer que lo importante en el estudio de la pobreza — desde una óptica multidimensional - no es solamente la cuantificación de los capitales con los que cuenta un determinado hogar, sino más bien la dinámica y articulación que existe entre ellos. Esto confirma la importancia de considerar el contexto cuando se estudia la pobreza, y la forma en que los hogares se organizan para mejorar sus condiciones de vida, por lo que no basta caracterizarlos a partir de la cuantificación de sus recursos o carencias. Y también demuestra que analizar el capital social de manera aislada no nos dice mucho acerca de los patrones de exclusión en términos económicos, humanos y sociales.

Lo anterior implica el reconocimiento de la complejidad inherente al momento de operacionalizar un concepto de naturaleza relacional, y la necesidad de sistematizar el análisis empírico ofreciendo tipologías inductivas que a su vez permitan la construcción de tipos ideales útiles en la comprensión de realidades concretas. Es decir, reconocer que la pobreza debe incluir elementos más allá del ingreso y considerar aspectos como el capital social implican necesariamente agregar retos metodológicos — como la combinación de trabajo cualitativo y cuantitativo-, así como teóricos —al hacer congeniar distintas conceptualizaciones-, a un tema de por sí difícil de sistematizar. Sin embargo, no queda otra opción si estamos comprometidos con conocer en mayor detalle las características y tipos de pobreza que existen para poder vincular este conocimiento con la generación de alternativas de solución desde la acción pública. 
En el caso de México, un enfoque de esta naturaleza permitirá entender cómo los programas de combate a la pobreza se integran a un tejido social previo, el cual muchas veces tiene implicaciones profundas para su implementación, lo que causa variaciones evidentes en términos del desempeño local de tales programas. Entender y dar cuenta de estas variaciones - más allá de incorporar un indicador numérico a la medición del CONEVAL — en el diseño de la política social es entonces el primer paso para plantear alternativas coherentes con los distintos contextos locales, especialmente en áreas urbanas marginadas como la presentada en este trabajo.

\section{Referencias}

Bebbington, Anthony, 2005, "Estrategias de vida y estrategias de intervención: el capital social y los programas de superación de la pobreza”, en Irma Arriagada (ed.), Aprender de la experiencia. El capital social en la superación de la pobreza, Santiago, Comisión Económica para América Latina y el Caribe (CEPAL), pp. 21-46.

Boltvinik, Julio, 2005, "Métodos de medición de la pobreza. Una tipología. Limitaciones de los métodos tradicionales y problemas de los combinados”, en J. Boltvinik, y A. Damián (coords.), La pobreza en México y el mundo. Realidades y desafios, México, Siglo XXI Editores/Gobierno del Estado de Tamaulipas, pp. 437-475.

Boltvinik, Julio, y A. Marín, 2003, "La canasta normativa de satisfactores esenciales de la Coplamar. Génesis y desarrollos recientes”, Comercio Exterior, núm. 53, México, pp. 473-484.

Bourdieu, Pierre, 1986, "The Forms of Capital”, en John G. Richardson (ed.), Handbook of Theory of Research of the Sociology of Education, Nueva York, Greenwood, pp. 241-258.

Burt, Ronald, 2005, Brokerage and Clousure: An Introduction to Social Capital, Boston, Oxford University Press.

Coleman, James S., 1988, "Social Capital in the Creation of Human Capital”, The American Journal of Sociology, núm. 94, Chicago, pp. 95-120.

CONEVAL, 2012, "Información por municipio", en Medición de pobreza multidimensional, México, Consejo Nacional de Evaluación de la Política de Desarrollo Social (coneval), disponible en <www.coneval.gob.mx>, consultado el 1 de febrero de 2012.

Coneval, 2010, Metodología para la medición multidimensional de la pobreza en México, México, Consejo Nacional de Evaluación de la Política de Desarrollo Social. 
Cortés, Fernando, D. Hernández, E. Hernández Laos, M. Székely, y H. Vera, 2002, Evolución y caracteristicas de la pobreza en México en la última década del siglo XX, México, SEDESOL (Serie Documentos de Investigación, 2).

Durston, John, 2003, "Capital social: parte del problema, parte de la solución, su papel en la persistencia y en la superación de la pobreza en América Latina y el Caribe”, en Raúl Atria, Marcelo Siles, Irma Arriagada, Lindon J. Robison, y Scott Whiteford, Capital social y reducción de la pobreza en América Latina y el Caribe: en busca de un nuevo paradigma, Santiago, CEPAL/Michigan State University, pp. 147-202.

Fuhse, Jan A., 2009, “The Meaning Structure of Social Networks”, Sociological Theory, vol. 27, núm. 1, Washington, pp. 51-73.

Gordon, Sara, 2008, "Pobreza urbana y capital social”, en Rolando Cordera, Patricia Ramírez Kuri, y Alicia Ziccardi (coords.), Pobreza, desigualdad social y exclusión social en la ciudad del siglo XXI, México, Siglo XXI/Instituto de Investigaciones Sociales-unAM, pp. 321-339.

Granovetter, Mark, 1973, “The Strength of Weak Ties”, American Journal of Sociology, Washington, vol. 78, núm. 6, pp. 1360-1380.

Kakwani, Nanak, y J. Silber, 2008, "Introduction: Multidimensional Poverty Analysis: Conceptual Issues, Empirical Illustrations and Policy Implications”, World Development, vol. 36, núm. 6, Washington, pp. 987-991.

Ley General de Desarrollo Social, 2004, México, sedesol, pp.1-18.

Lewis, Oscar, 1961, Antropología de la pobreza. Las cinco familias, México, Fondo de Cultura Económica.

Lin, Nan, 1999, "Social Networks and Status Attainment”, Annual Review of Sociology, vol. 25, núm. 1, Washington, pp. 467-487.

Lomnitz, Larissa, 2006, Cómo sobreviven los marginados, México, Siglo XXI.

Martínez, Irma, J. Trevińo, y M. Gómez, 2009, Mapas de pobreza y rezago social. Área Metropolitana de Monterrey, Monterrey, Consejo de Desarrollo Social.

Millán, René, y S. Gordon, 2004, "Capital social: una lectura de tres perspectivas clásicas”, Revista Mexicana de Sociología, vol. 66, núm. 4, México, pp.711-747.

Nahapiet, Janine, y S. Ghoshal, 1998, "Social capital, intellectual capital, and the organizational advantage", Academy of Management Review, vol. 23, núm. 2, Nueva York, pp. 242-266. 
Narayan, Deepa, 1999, Bonds and Bridges : Social Capital and Poverty, Washington, World Bank.

Nélida, Perona, y G. I. Rocchi, 2007, "Vulnerabilidad y exclusión social. Una propuesta metodológica para el estudio de las condiciones de vida de los hogares", Kairos. Revista de Temas Sociales, núm. 8, pp. 1-15.

Noll, Heins-Herbert, 2010, "The Stiglitz-Sen-Fitoussi-Report: Old Wine in New Skins? Views from a Social Indicators Perspective”, Social Indicators Research, vol. 102, núm. 1, pp. 111-116.

Portales, Luis, y A. Ruiz, 2013, "Amilpa: un ejemplo de participación ciudadana en acción colectiva para la obtención de servicios públicos”, Espacios Públicos, vol. 16, núm. 36, Estado de México, pp. 117-143.

Portes, Alejandro, 1998, "Social Capital: Its Origins and Applications in Modern Sociology", Annual Review of Sociology, vol. 24, núm. 1, Washington, pp.1-24.

Portes, Alejandro, y P. Landolt, 2000, "Social Capital: Promise and Pitfalls of its Role in Development”, Journal of Latin American Studies, vol. 32, núm. 2, Cambridge, pp. 529-547.

Portes, Alejandro, y P. Landolt, 1996, "The Downside of Social Capital”, The American Prospect, mayo-junio, Washington, pp. 18-22.

Putnam, Robert, 1995, “Bowling Alone: America’s Declining Social Capital”, Journal of Democracy, vol. 6, núm. 1, Washington, pp. 65-78.

Putnam, Robert, 1993, "The Prosperous Community. Social Capital and Public Life", The American Prospect, vol. 4, núm. 13, Washington, pp. 1-11.

Robison Lindon, J., M. E. Siles, y A. A. Schmid, 2003, "El capital social y la reducción de la pobreza: hacia un paradigma maduro", en Raúl Atria, y Marcelo Siles (comps.), Capital social y reducción de la pobreza en América Latina y el Caribe: en busca de un nuevo paradigma, Santiago, Comisión Económica para América Latina y el Caribe/Michigan State University, pp. 51-114.

Rodríguez, Héctor, y M. Kinto, 2010, "Crecimiento poblacional y calidad de vida en el Área Metropolitana de Monterrey, 1990-2005”, en L. Palacios (ed.), Cuando México enfrenta la globalización. Permanencias y cambios en el área metropolitana de Monterrey, Monterrey, Universidad Autónoma de Nuevo León, pp. 47-66.

Rojas, Mariano, 2010, "Mejorando los programas de combate a la pobreza en México: del ingreso al bienestar”, Perfiles Latinoamericanos, núm. 35, México, Flacso México, pp. 35-39. 
Sen, Amartya, 1981, "Public action and the quality of life in developing countries", Oxford Bulletin of Economics and Statistics, vol. 43, núm. 4, Oxford, pp. 287-319.

Siles, Marcelo E., L. J. Robison, y S. Whiteford, 2003, “Introducción”, en Raúl Atria, Marcelo Siles (comps.), Capital social y reducción de la pobreza en América Latina y el Caribe: en busca de un nuevo paradigma, Santiago, Comisión Económica para América Latina y el Caribel Michigan State University, pp. 1-20.

Stiglitz, Joseph, A. Sen, y J.-P. Fitoussi, 2008, Report by the Commission on the Measurement of Economic Performance and Social Progress, París, Sustainable Development.

Svendsen, Gert T., y G. L. Haase Sevendsen, 2009, “The troika of sociology, political science and economics", en Gert Tinggaard Svendsen (ed.), Handbook of Social Capital. The Troika of Sociology, Political Science and Economics, Cheltenham, Edward Elgar, pp. 1-13.

Townsend, Peter, 2003, "La conceptualización de la pobreza”, Comercio Exterior, vol. 53, núm. 5, México, pp. 445-451.

Vigil, James Diego, 2003, "Urban Violence and Street Gangs", Annual Review of Anthropology, vol. 32, núm. 1, Palo Alto, pp. 225-242.

Wellman, Barry, y S. Wortley, 1990, "Different Strokes from Different Folks : Community Ties and Social Support", The American Journal of Sociology, vol. 96, núm. 3, Chicago, pp. 558-588.

Woolcock, Michael, 1998, "Social capital and economic development: Toward a theoretical synthesis and policy framework", Theory and Society, núm. 27, pp. 151-208.

Woolcock, Michael, 2001, “The Place of Social Capital in Understanding Social and economics outcomes", Canadian Journal of Policy Research, vol. 2, núm, 1, Toronto, pp. 65-88.

World Bank, 2006, Social Capital for Development, Washington, World Bank, disponible en <http://www1.worldbank.org/prem/poverty/scapital/>, consultado el 29 de agosto de 2011.

Ziccardi, Alicia, 2008, "Ciudades latinoamericanas: procesos de marginalidad y de exclusión social”, en Rolando Cordera, Patricia Ramírez Kuri, y Alicia Ziccardi (coords.), Pobreza, desigualdad social y exclusión social en la ciudad del siglo XXI, México, Siglo XXI, pp. 73-91.

Recibido el 16 de febrero de 2012.

Aceptado el 9 de julio de 2014. 